Modeling of Thinking and the Mind 


\title{
Modeling of Thinking and the Mind
}

N.M. Amosov

\author{
Translated by \\ Leo Finegold \\ San Diego Mesa College \\ for Scripta Technica, Inc.
}

Translation Editor

Lawrence J. Fogel President, Decision Science, Inc.

San Diego, California 


\section{MODELING OF THINKING AND THE MIND}

(C), 1967 by Spartan Books.

Softcover reprint of the hardcover 1st edition 1967

All rights reserved. This book or parts thereof may not be reproduced in any form without permission from the publisher. Library of Congress Catalog Card Number 67-27007

Originally published under the title of MODELIROVANIYE MYSHLENIYA I PSIKHIKI by Naukova Dumka Press, Kiev, 1965

Sole distributors in Great Britain, the British Commonwealth, and the Continent of Europe:

Macmillan and Company, Ltd.

4 Little Essex Street

London, W. C. 2

ISBN 978-1-349-00642-7 ISBN 978-1-349-00640-3 (eBook)

DOI 10.1007/978-1-349-00640-3 


\section{Preface}

The advent of information theory and computer technology has focused the attention of science upon the functional aspects of mechanism in a new way. The hardware becomes of interest in terms of its information processing attributes. Systems are studied as interrelated configurations of information processing elements. The reductionist problem becomes one of finding the appropriate code rather than simply locating the site of action.

This orientation has been embraced by the scientific community within the Soviet Union in the name of their Cybernetic Program which attempts to generate "Scientific Government" (presuming, of course, the sanctity of some higher-level goal). This philosophic viewpoint has probably had greatest impact in the fields of biology and psychology where the concern is with the nature of thinking in terms of properties and mechanisms.

This book offers a survey of the state of the art as seen by an eminent scientist from his unique vantage point within the Soviet spectrum of activities. After examining fundamental considerations germane to the philosophy of science and the development of mathematical models, he proceeds to consider information processing at increasing levels of complexity within the living organism. Detailed considerations are devoted to intracellular homeostatic mechanisms, with the explicit recognition that homeostasis must be modifiable if there is to be learning. He proceeds to intercellular mechanisms, such as the endocrine system and the nervous system, finally focusing attention upon the information processing aspects at higher levels of human behavior.

Granted, some of the concepts presented in these latter chapters might be called speculative, they do, however, represent the direction of thought in this area of significant interest. Amosov apparently realizes the importance of this overall perspective in terms of computer realizations of artificially intelligent systems in the near future. He concludes, "It seems to me that it's simply unthinkable to plan the building of a new society without quantitative consideration of the psychology of its members." This demonstrates a new perspective in Soviet philosophy, a freedom of the scientist to offer precepts to those who control the Government. Their 
acceptance of this represents a new level of sophistication which might indeed result in dramatic changes in future Soviet policy with respect to science, internal politics, and even international development.

Throughout, there has been an attempt toward direct translation of Soviet terminology, except where recognized idioms are used. This book represents a worthy attempt to blend the common interests of biology, psychology, and engineering. It provides material of interest to the entire spectrum of those interested in science, technology and its impact upon the future of society.

LEO FINEGOLD, M. A.

LAWRENCE J. FOGEL, PH. D. 


\section{Contents}

PREFACE v v

INTRODUCTION

1. SOME GENERAL ASSUMPTIONS OF CYBERNETICS 1

2. THE EVOLUTION OF PRE-HUMAN LIVING SYSTEMS $\begin{array}{lc}\text { AND THEIR PROGRAMS } & 34\end{array}$

3. BASIC PROGRAMS OF HUMAN BEHAVIOR

4. CONSCIOUSNESS, THINKING, CREATIVITY 114

5. MODELING OF MENTAL FUNCTIONS 147

$\begin{array}{lr}\text { CONCLUSION } & 185\end{array}$

$\begin{array}{lr}\text { INDEX } & 189\end{array}$ 


\section{Introduction}

Problems of thinking and of the human mind have attracted the interests of thinkers throughout history. Philosophers of antiquity, naturalists of the Middle Ages, the erudite of the Renaissance, and scholars thereafter have been concerned with just these problems. Today the mind is being studied by neurophysiologists, by psychologists, by psychiatrists, and by philosophers of various disciplines.

Marx, Engels, and Lenin explicitly define the materialistic essence of thought processes in a philosophic way: The brain is the substrate of thought and man's behavior is contingent upon it. However, even at the present time, some scholars adhering to idealistic concepts, do not agree with this thesis. They assert that nonmaterial forces of one form or another function in thinking, as for example, in telepathy. To declare such observations to be fiction would be unscientific. Insofar as there are doubts as to the methodological purity of such experiences, it is necessary to verify them employing the achievements of modern technology: It is possible that some factors will be confirmed and information transfer will be registered without the employment of the usual material agents. Of course, this would not in any way, by itself, invalidate the concept of materialism. It would simply be necessary to look for new physical forces which might be manifested only at the level of highly organized living creatures. We cannot rule out such a situation since it would be a mistake to view the processes of life merely as an interaction of such relatively large particles as atoms and molecules. If physics admits the existence of even smaller elements of matter than these elementary particles, it is certain that these too participate in living systems. It is possible that the organism might be that very indicator which allows one to discover the activity of such particles under some particular conditions.

I admit the possibility of "wireless" transmission of psychic events, but I suspect that their significance in human behavior is not great. However, this view is relevant only so long as the authenticity of telepathy still remains to be confirmed.

But this is what is important. It is trivial to acknowledge the brain as the material substrate of the psyche. One must 
demonstrate the mechanism of its activity. In what way does it direct the behavior of man? When this is done, many doubts will disappear as will the desire to make use of miracles as an explanation for some of our actions. This is a very difficult task. The brain consists of many billions of distinct nerve cells, and in turn, each of these cells consists of a huge number of molecules of different types. We no longer doubt that human actions are determined by molecular and cell interrelationships. But a final, all-embracing proof that this is so will require models which represent the structure of the brain and whichwill reproduce its function, that is the behavior of man.

Some philosophers assume that the total activity of a very complicated system cannot be reduced to a composite function of its more simple elements; I believe they are mistaken. Such a point of view would make it impossible in principle to develop an artificial model which would reproduce the basic functions of a complex system. To state this is tantamount to declaring: "The brain is material, thinking is its function, but it is impossible to understand its activity." The highest form of understanding of a system is the reproduction of its structure and function, if not in full, then at least in its main manifestations. Man is fully capable of such an achievement.

Until very recently it was impossible to even dream of working models of complex systems consisting of an astronomical number of elements. The "details" out of which man constructed his models were too large and "dead," being incapable of change. The situation today has changed, or at least is changing.

Technology is giving us new elements: first, electronic tubes, then semiconductors, now thin films and microminiature circuits. The construction of artificial working systems of great complexity, of high reliability, and of colossal speed has become a real possibility. In principle, it has become possible to create a model approaching the brain in complexity. The crux of the problem is how to arrange the elements in it so that by their interaction, the model would more or less completely duplicate the living organism. In other words, the problem is to create machines which have the programs equivalent to that of the brain.

Unfortunately, there are still many impediments in this direction. First, we have a poor understanding of the structure and function of the brain. Physiologists have given us only very gross models depicted by diagrams and in terms of words. One cannot expect more for the time being since their means of modeling - of comprehension - is quite limited. In reality it is exceedingly difficult to gain insight into the highly complex schematic of the brain, this apparatus consisting of billions of elements and of a much greater number of connections between them, all contained in a volume which hardly exceeds a cubic decimeter. One cannot expect to obtain schematics of the brain and a description of its programs in the immediate future. 
Does this then mean that modeling of human thought and of the mind must be postponed for an indefinite time? Does this mean that we must acknowledge the participation of "higher forces" or that modeling is, in principle, impossible because of qualitative peculiarities of the mind which, therefore, apparently cannot be reduced to computation?

There is no justification for such pessimism. It does not pay to wait for the anatomists and physiologists to decode the brain. Science, has an alternative - to study human behavior as an integrated system and model it by means of technological elements without attempting to maintain anatomical or physiological similarity to the components of the brain. Nature has developed much that is interesting, but human genius competes quite successfully with her.

However, can we now begin to model man's mind as an integrated system? Do we have sufficient data? Unfortunately, the answer is no.

Two scientific disciplines study human behavior: psychology (which embraces the study of normal people) and psychiatry (that of abnormal people). Both of these sciences are at that stage of development where, for the time being at least, they cannot offer material for modeling. The fact of the matter is that a necessary condition for the creation of any model is computation, that is quantitative regularity. Up to the present time, these sciences have almost exclusively been governed by qualitative concepts. They describe their systems by words, by simple graphs, and only very rarely by numbers (and this only in very special cases).

Of course, psychologists and psychiatrists are not responsible for this. Human behavior is very complex and varied. It is determined by a multitude of the most diverse influences of external and internal origin. Until very recently, the variety of human behavior was discerned by sense organs only and was described in verbal terms. Instruments for sensing and recording were extremely limited.

Now the possibilities for an objective study of the human mind have substantially increased. Complex technological systems, universal or special purpose computers are capable of receiving and retaining extremely varied information over protracted periods of time. Methods for processing this information exist which permit rapid selection of the required data or for the discovery of new information. It is true that as yet there is no justification for exaggerating the potential capability of these machines relative to logical operations, but, with each passing year, this capability is growing. Machines can already actively retain more data relative to more or less narrow problems than can man. And what is more important, machines can manipulate this data far more quickly.

This permits one to express the essence of man in terms of a set of numbers at whatever level of detail is desired. Of course, 
we are still very far from that time when a numerical model will be complete, but its probability, that is, its degree of coincidence with the original, can already be significantly higher than that of the probability of those models which are stored in our memory and which are expressed by words.

Initially, of course, it is necessary to develop a method of investigation which rests upon appropriate technical mechanisms and codes which permit portrayal of the findings in terms of numbers. Programs are also necessary for the processing of such numerical data. All of this, taken together, can be called "contemporary instrumental" psychology or possibly "cybernetic" psychology.

To begin working towards the creation of this new direction in science, some hypotheses about the mechanism or program of human behavior is essential. Every investigation must have a goal which has been derived from one or more conjectures. Even with perfect technology, one cannot exhaustively study everything that is sensed. Man is too complex to expect rapid discovery of valuable data. Besides, psychology and physiology have already accumulated enough facts to offer quite a few assumptions which permit representation of the human mind in broad outline. Further investigations on a new level, resting on quantitative data, must substantiate some of these assumptions and refute others.

In general, an hypothesis about thinking and human behavior is essential. An hypothesis is a model which is expressed in words or schematics. It may be broad-embracing many aspects of behavior of the original system, or narrow-describing particular problems. It may be general or detailed. Thus, for example, the task of a physiological hypothesis, which interprets the meaning and reciprocal relationship of different anatomical and functional sections of the brain, is intended to characterize the behavior of man as an integrated system. I have already said, that the time has not yet arrived for such a broad and deep hypothesis, and I doubt that it will be possible to construct such an hypothesis utilizing conventional means. However, even now, many particular hypotheses about the influence of various anatomical formations of the brain on human behavior are sufficiently validated and are suitable for use.

One can compose an hypothesis in a purely "informational" way - a psychological construct which does not rest on the anatomy of the brain but which has as a base only experience according to which some structural scheme of internal relations is created which exhibits the required effect in a manner most similar to that of human behavior. I believe it justifiable to say that such a fictional man functions as a real one.

In this book an attempt is made to state a similar hypothesis. It has a most direct bearing on simulation insofar as:

1. every hypothesis is a model of a system which is expressed 
in different codes and mechanisms. We have tried to make our model broader and more detailed;

2. our model is algorithmic, that is, from it one can create programs for computers or technical specifications for the development of special mechanisms which can reproduce various aspects of the hypothesis in an operating artificial system. In other words, these are algorithms for heuristic modeling. Of course, in order to acquire them it is necessary to be given sufficient data to quantitatively characterize the hypothetical interrelations.

Heuristic simulation is as valid a method for gaining knowledge as are experimental investigations. Experiments yield facts from which we draw conclusions about the system behavior; by these conclusions the hypothesis is verified. We can reverse this procedure: given some fact, and on this basis construct a hypothesis of behavior in order to derive new facts. If these coincide with the data of subsequent experiments, the hypothesis is verified.

The general task which confronts contemporary life science is that of increasing its precision: It is necessary to depart from qualitative descriptions and proceed to quantitative models. All this is relevant even to psychology. Its task is to create models of human behavior. Of course, these will be probabilistic and numerous. But techniques exist which are suitable for the selection and retention of appropriate ones. It is necessary to create methods of testing the mind which permit its quantitative evaluation so that there is the possibility of relating man to various models. Only after this will we be able to calculate the behavior of an individual and, what is still more important, of a group of different people under specified conditions. I am convinced that without such computation in the future, it will become impossible to plan important governmental policies. 